\author{
RAFAEL TEMES CORDOVEZa \\ MOISÉS SIMANCAS CRUZ \\ MARÍA PILAR PEÑARRUBIA ZARAGOZAc \\ ALICIA GARCÍA AMAYA
}

\title{
APLICACIÓN DE MODELOS DIGITALES A LA DETERMINACIÓN DEL VALOR DE VISTAS AL MAR EN LAS ÁREAS TURÍSTICAS DE LITORAL
}

\begin{abstract}
RESUMEN
El uso masivo de datos geográficos requiere de información cartográfica. Los modelos digitales derivados del procesado de datos LIDAR (Light Detection and Ranging) se plantean como fuentes de información, entre otros campos, en el análisis del paisaje urbano. Así, su aplicación permite la valoración de las líneas de horizonte de un observador situado en un entorno urbano concreto. Esta es una cuestión especialmente relevante en las áreas turísticas de litoral, donde, por ejemplo, las tarifas son distintas entre habitaciones de un hotel con o sin vistas al mar. El objetivo de este trabajo fue comparar el resultado de las líneas de horizonte obtenidas desde un mismo punto de observación a partir de dos métodos de cálculo diferentes. El Método 1 utilizó el Modelo Digital de Superficie (MDS) obtenido a partir de archivos.LAZ de $2 \times 2 \mathrm{~km}$ de extensión (densidad de 0,5 puntos /m2), procedentes del Instituto Geográfico Nacional (IGN) combinado con la delimitación de huella de edificios según Catastro. El Método 2 utilizó el atri-

a Departamento de Urbanismo. Universitat Politècnica de València. Camino de Vera s/n, 46022 Valencia / Grupo de investigación ReinvenTUR. Universidad de La Laguna. rtemesc@urb.upv.es. https://orcid.org/0000-0002. $5604-4892$

b Departamento de Geografía e Historia. Universidad de La Laguna. Campus de Guajara. Apartado 456. 38200 La Laguna. S/C de Tenerife / Grupo de investigación ReinvenTUR. Universidad de La Laguna. msimancas@ull. edu.es. https://orcid.org/0000-0002-0337-0424

c Departamento de Geografía. Universitat de València. Av. Blasco Ibañez, 28. 46010 Valencia / Grupo de investigación ReinvenTUR. Universidad de La Laguna. M.Pilar.Penarrubia@uv.es

d Universitat Politècnica de València. Camino de Vera s/n, 46022 Valencia. alicia@garciaamaya.com https:// orcid.org/0000-0003-3114-4712
\end{abstract}

Fecha de recepción: 4/7/19. Fecha de aceptación: 14/12/19. 
buto de altura de edificios proveniente del Catastro sobre el Modelo Digital del Terreno (MDT) como referencia. El ámbito de análisis fue el de Puerto de la Cruz (Tenerife, Canarias). Los resultados obtenidos inicialmente nos demuestran que, a la hora de realizar un análisis de visibilidad preciso, logramos mejores resultados con los archivos LIDAR que con la combinación entre un MDS y la volumetría edificada del Catastro. También se constata la existencia de algunos errores de clasificación de puntos en los archivos LIDAR que obligan a utilizar un método intermedio para mejorar los resultados.

Palabras clave: espacio turístico de litoral, Catastro, LIDAR; Modelo Digital de Superficie, Modelo Digital del Terreno

\section{APPLICATION OF DIGITAL MODELS TO DETERMINE THE VALUE OF SEA VIEWS IN COASTAL TOURIST AREAS}

\section{Abstract}

The proliferation of geographic information mass capture systems requires the development of methods and strategies for their automatic processing. LIDAR (Light Detection and Ranging) devices are a clear example of this evolution. It is used in different fields: urban planning, hydrology, environment and, of course, in tourism as a synthesis of all of the above. Landscape is often one of the main resources in tourist areas.

The objective of this work was to compare the result of skylines obtained from the same observation point from two different calculation methods. Method 1 used the Digital Surface Model (DSM) obtained from LAZ files of $2 \times 2 \mathrm{~km}$ extension (density of 0.5 points $/ \mathrm{m} 2$ ), coming from the National Geographic Institute (IGN) combined with the delimitation of the footprint of buildings according to Cadastre. Method 2 used the building height attribute from the Cadastre on the Digital Terrain Model (DTM) as a reference. The scope of analysis was Puerto de la Cruz (Tenerife, Canarias).The results we initially obtained show us that, when carrying out an accurate visibility analysis, we obtain better results with LIDAR files than with the combination of a MDS and the built volumetry of the Cadastre. However, we also found some errors in the classification of points in the LIDAR files that require the use of an intermediate method to improve the results. This communication is part of a broader investigation that focuses on the analysis and description of the urban form of coastal tourism in the Canary Islands.

Key Words: coastal tourist space consolidated, Cadastre, LIDAR, Digital Terrain Model, Digital Surface Model

\section{INTRODUCCIÓN}

La explotación de los datos incorporados en sistemas de big data, open data o la reutilización de la información pública disponible suponen una oportunidad de mejora para los gestores de los destinos turísticos. Estos datos, convenientemente recogidos, almacenados, procesados e interpretados, aportan el conocimiento que los actores turísticos necesitan para 
tomar decisiones adecuadas a las diferentes situaciones (Peñarrubia, 2018). De acuerdo con Ivars et al. (2017), "la inteligencia para un destino turístico [...] consiste en seleccionar, conforme a sus características, los datos más valiosos para el gestor y empresariado local. Su análisis y gestión integrada en base a indicadores clave, permitirá obtener ventajas competitivas que sustenten líneas de acción inteligentes”. La monitorización y digitalización de los destinos turísticos y de los turistas, han generado un incremento exponencial en la producción de datos. Desde este punto de vista, los datos, convertidos en información, adquieren valor al aplicarse en los procesos de toma de decisiones en los ámbitos privados y públicos, convirtiéndose en conocimiento. Ello reduce la incertidumbre que implica un entorno cambiante y competitivo con el turístico.

La adopción del enfoque tecnológico de los destinos turísticos inteligentes ha impulsado un proceso de creación, recogida y gestión de datos que se incorporan a Sistemas de Inteligencia Turística, atendiendo a múltiples necesidades y demandas actuales de información del sector (Celdrán, Mazón y Giner, 2018). En esta línea, se ha desarrollado una analítica de datos, que engloba todos los procesos matemáticos y estadísticos que permiten convertir los datos brutos en información práctica. Lo mismo ha sucedido con la posibilidad de mejorar la relevancia, oportunidad y puntualidad de los productos estadísticos ya ofrecidos por las estadísticas públicas con otras fuentes de información complementarias privadas, como los dispositivos de control de tráfico, los datos de posicionamiento de dispositivos móviles o la actividad de las tarjetas de crédito durante un viaje (Cortina, Izquierdo, Prado y Velasco, 2016).

Este trabajo surge en este contexto y pretende aportar ideas que orienten los procesos de adopción de estrategias basadas en el análisis y puesta en valor de los datos turísticos, así como su gestión inteligente. Para ello, el principal objetivo del estudio es valorar la utilidad de algunas fuentes de información pública derivadas del uso de las tecnologías de análisis espacial. En esta línea hay trabajos previos que ponen en valor la información del Catastro para el análisis de las trasformaciones urbanas (Temes, 2008; Santos-Preciado, 2015; Moya, 2017) o que emplean la tecnología LIDAR para valorar cambios en los edificios (Hermosilla et al, 2009; Francés, 2015; Santos-Pérez, 2015; Pérez et al, 2015). Con el fin de dotar de una dimensión aplicada hemos escogido un entorno turístico litoral consolidado, como es el Puerto de la Cruz (Tenerife, España). El motivo de la elección se justifica por dos razones fundamentales. Por un lado, por su condición geográfica de destino turístico abierto al mar hacia el norte, con una leve inclinación, que lo convierte en uno de sus recursos más característicos y valorados por los visitantes. Estas vistas del Océano Atlántico se complementan hacia el interior de la isla (el sur), con la presencia del Valle de La Orotava y, sobre todo, el perfil del Teide. Por otra, se trata de un área turística inmersa en un proceso de regeneración turística a través de su Plan de Modernización, Mejora e Incremento de la Competitividad de Puerto de la Cruz, aprobado por el Decreto 107/2015, de 22 de mayo; nuestra aportación 
puede servir para la posible definición de propuestas de reordenación urbanística o renovación física y funcional (modelo de negocio) de algunos alojamientos turísticos.

\section{Planteamiento del problema:}

LA IMPORTANCIA DE LAS VISTAS AL MAR EN LA RENOVACIÓN DE LOS ESTABLECIMIENTOS DE ALOJAMIENTO TURÍSTICO

La Sentencia de 31 de mayo de 2005 de la Sala Tercera, de lo Contencioso-Administrativo del Tribunal Supremo sentó un precedente en relación a la patrimonialización del paisaje: anuló el Estudio de Detalle de la parcela $\mathrm{n}^{\circ} 5$ del Polígono Racó II, promovido por la empresa Florazar, S.A., ubicado en la playa del Racó de Cullera (Valencia, España). El Estudio de Detalle fue aprobado definitivamente por acuerdo del Pleno del Ayuntamiento de Cullera de fecha 27 de mayo de 1997, así como la concesión de licencia de obras con fecha de 16 de marzo de 1998. Dicho Estudio de Detalle modificó la forma del bloque, haciéndolo más estrecho en una zona (su crujía pasó de 13,50 m. a 12,00 m.), reduciendo su longitud (seis metros) y aumentando su ancho en otra parte (pasó de 13,50 m. a 16,55 m.). Este cambio causó a la Comunidad de Propietarios del edificio Casa d'Anna un perjuicio por pérdida de parte del soleamiento y por la reducción de las vistas al mar en dos puntos: por un lado, desde el vértice del Casa d'Anna más próximo al mar, donde se redujo el ángulo de visión del mar de los 32 grados a los 20 grados, y por otro, desde el punto central del bloque de la finca paralelo a la playa, donde se rebajó el ángulo de visión de 23 grados a 20 grados.

El Tribunal Supremo reconoció el derecho de la Comunidad de Propietarios a la indemnización de ese perjuicio de pérdida de la calidad de las viviendas. Cabe destacar que mientras los peritos de la consultora Tinsa, contratados por la empresa Florazar, S.A. estimaron que la indemnización debía ser de entre 245.884 y cero euros, dada la "dificultad de establecer un valor", el informe pericial de la Universitat Politècnica de València que aportó la Comunidad de Propietarios la cifró en más de seis millones de euros en función de los costes de derribo que serían necesarios para restituir los ángulos de visión. Finalmente, la perita designada por la sala de lo contencioso-administrativo del Tribunal Superior de Justicia de la Comunidad Valenciana a instancia del Ayuntamiento de Cullera la fijó en 37.848 euros. Esta cuantía resultó de la estimación del valor de pérdida de cada grado sexagesimal de visión era de 93,46 euros. Para ello, la perita superpuso los planos originales con los ejecutados. Así, fijó una indemnización de 280 euros a la totalidad de las 99 viviendas afectadas del bloque I de la Urbanización Casa d'Anna (edificio paralelo al mar) y de 1.121 euros a las 9 del bloque II. La determinación de esas cuantías se comparó el precio que las viviendas que están en primera línea de playa, con las de segunda línea tenían en el año 1997.

Por su parte, la sentencia de la Sala Contencioso-Administrativo del Tribunal de Justicia de Canarias de 10 de octubre de 2002 anuló la licencia otorgada por la Comisión Municipal de Gobierno del Ayuntamiento de Las Palmas de Gran Canaria (Canarias, España) al 
Ministerio de Educación para la construcción de una biblioteca pública del Estado en un solar contiguo al edificio San Telmo. Dicha anulación se basó en justificar que la licencia fue concedida sin haberse redactado el Plan Especial exigible y que se cambiaron extremos en el Proyecto autorizado sin modificación de la ordenanza, de manera que se alteraba la determinación establecida en el Plan General de Ordenación Urbana respecto del sistema general cultural administrativo y de espacios libres, se vulneraba la zonificación en cuanto a zona verde con privación de $817 \mathrm{~m}^{2}$ y se ubicaba el edificio en un emplazamiento totalmente distinto. La Comunidad de Propietarios presentó una demanda de responsabilidad patrimonial contra el Ayuntamiento de Las Palmas por la lesión patrimonial por pérdida de las vistas al mar. El informe pericial estimó que la depreciación por esa pérdida tenía un valor máximo de 1.200 euros $/ \mathrm{m}^{2}$; a partir de esa cuantía, la perita aplicó porcentajes y coeficientes para adaptarlo a las características de cada inmueble, cuantificando exhaustivamente la pérdida del valor de cada vivienda del edificio. Ese sería el daño permanente cuantificado en el año 2009 y para actualizarlo a diciembre de 2011 aplicó los coeficientes del Instituto Nacional de Estadística. De ahí se obtuvo el valor de depreciación provocado por la construcción de la Biblioteca actualizado al año 2011 que calculó en 3.456.530,16 euros. A su vez, la perita calculó el valor de la pérdida día a día multiplicando esta cantidad por 13 años (transcurridos desde la construcción en 1998 y el año de cálculo 2011) y dividiéndolo por los 100 años de vida útil del edificio.

Lo interesante de ambas sentencias es que ponen de manifiesto el "derecho a las vistas" de un edificio conforme a lo establecido en el correspondiente instrumento de planeamiento urbanístico. No obstante, hay que tener en cuenta que el aprovechamiento urbanístico de las parcelas de ambas Comunidades de Propietarios demandantes no incluía un "derecho de vistas al mar", en cuanto el planeamiento urbanístico no otorga o priva a una parcela o edificación del mismo, sino un espacio libre que, de haberse respetado, no hubiese obstaculizado las vistas. Considerando que el mar constituye una parte importante del imaginario de las áreas turísticas de litoral, la aplicación del "derecho al mar" pone en valor la ubicación geográfica de los alojamientos turísticos, en general, y las vistas del mar, en particular. Esto hace necesario no sólo que la temática paisajística se incluya en los procesos de planificación de las áreas turísticas de litoral (De Aranzabal, Schmitz y Pineda, 2009; Dos Santos, 2011; Santos, Alfonso y Muñoz, 2016), dado que hasta el momento ha estado prácticamente ausente (Vera et al., 2011), sino disponer de técnicas, metodologías e instrumentos adecuados que aporten los datos necesarios para la toma de decisiones en esta materia basados en el análisis espacial.

La actividad turística de litoral se articula en torno al producto "sol y playa". Así, las externalidades de la primera línea de costa la han situado como el principal atractivo para el mercado turístico e inmobiliario. Esto ha provocado un importante proceso de urbanización de las franjas costeras. Así, se establece una relación entre el valor y el precio de un deter- 
minado elemento del área turística con su proximidad de una serie de recursos paisajísticos (Villar, 2013).

Desde esta perspectiva, la dimensión economicista del paisaje que plantean los modelos hedónicos adquiere un especial sentido en el caso de la urbanización turística (Villar, 2013). En esta línea, diversos trabajos han puesto de relieve la importancia de las vistas hacia espacios acuáticos como el mar o los lagos (Legget y Bockstael, 1999; Gupta et al, 2009; Schultz y Waltert, 2009). Otros estudios han abordado el cálculo del precio hedónico de los edificios basado en una serie de factores como las vistas, calidad ambiental, localización, etc. (Sander y Manson, 2007; Cavailhès et al. 2009; Hamilton y Morgan, 2010). Asimismo, destacan algunos trabajos que utilizan las cuencas visuales para analizar el número de edificaciones visibles como factor de decisión en el planeamiento urbano (Danese, Nolé y Murgante, 2009; Otero, Varela, Mancebo y Ezquerra, 2009; Danesa, Nolè y Murgante, 2011).

Este interés se debe a que determinados recursos visuales como el mar otorgan un valor añadido en el mercado inmobiliario del suelo y la vivienda, así como en los precios de los alojamientos turísticos. De esta manera, el mar adquiere la condición de recurso, que es aprovechado por las viviendas y establecimientos de alojamiento, poniéndolo en valor y vinculándolo a una estrategia turística. Ello convierte "las vistas al mar" en una "amenidad" indirecta meramente perceptual, pero cuya mercantilización continúa siendo muy rentable a través de un producto visual integral (More, Stevens y Allen, 1988). Así, el "poder ver" se traduce en diferentes rentabilidades económicas. Así, la cantidad y la calidad de las vistas al mar se traducen en un sobrevalor del bien inmueble. Sirva como ejemplo, la perita del citado informe del edificio San Telmo utilizó un método de comparación con estudios de mercado para determinar unos valores concretos distinguiendo una vivienda en edificio residencial sin vistas al mar con valor de 2.196 euros $/ \mathrm{m}^{2}$ y las viviendas con vistas al mar 3.414,96 euros/ $\mathrm{m}^{2}$. Si bien podemos entender que las vistas suponen un "valor" que, de manera general, se patrimonializa finalmente en el costo de un inmueble de uso residencial, la situación es mucho más clara y directa en el caso de la actividad turística: la fijación de tarifas distintas entre habitaciones de un hotel con o sin vistas al mar inciden en los procesos de pricing, al tener un precio más elevado, con el consiguiente impacto sobre los indicadores del negocio turístico y de la rentabilidad empresarial (ingresos por habitación disponible, beneficio en bruto por habitación disponible, total de ingresos por habitación disponible, ingresos de habitaciones/número de habitaciones ocupadas, etc.); de esta manera, las habitaciones de un hotel con vistas al mar se comportan como variables parametrizable en los procesos de revenue management. Lo mismo sucede con aquellos establecimientos hoteleros que han instalado piscinas con solarium, bares, restaurantes, terrazas, etc. en sus azoteas, hasta ese momento concebidas como espacios residuales y meras cubiertas. El propósito es aprovechar el valor de estas estructuras a través de su potencial generador de vistas paisajísticas a lo largo de espacio turístico mediante el disfrute de sus vistas, atardeceres, etc. Ello explica que los patrones de ocupación urbana en las áreas turística del litoral por parte del sector inmobiliario y aloja- 
tivo reflejen una búsqueda de la posibilidad de disfrute perceptual del mar. De esta manera, la lógica de la centralidad pierda interés en los destinos turísticos, ganando otros elementos como las perspectivas sobre el mar (Antón, 1998).

En este punto surgen los conflictos. La intensa actividad urbanizadora del frente litoral ha obligado a ocupar segundas y terceras líneas, así como a la modificación de alturas, volúmenes y formas de la edificación. Ello ha provocado la pérdida de vistas de terceros. Esto genera la necesidad metodológica de disponer de técnicas ad hoc y herramientas de anticipación de los escenarios urbanos con suficiente precisión y calidad que permitan identificar y valorar los perjuicios que una determinada operación urbanística podría provocar en relación con la ocultación de vistas o efecto pantalla de un edificio respecto a otro.

\section{Metodología y FUentes de INVESTigación}

Este trabajo se inserta dentro de los estudios sobre paisajes visuales. Estos pueden ser definidos como la articulación de las propiedades visuales generadas por (o asociada a) una configuración espacial específica (Llobera 2003). Así, los análisis de visibilidad son llevados a cabo por una cuenca visual teórica, entendida como "el conjunto de todas las localizaciones o puntos del territorio que son visibles desde un punto de observación específico, dada una distancia máxima de visión, y en base únicamente a la topografía" (García Sanjuán, 2006). Su determinación conlleva un análisis sobre el territorio cuyo resultado determina las zonas visibles y no visibles para uno o varios puntos de observación, dependiendo, entre otros factores, de la distancia máxima de estudio, la altura de observación, la del punto observado y el ángulo de visión de estos puntos (Pellicer et al., 2014). Estas cuencas dan, por agregación, un mapa de zonas de influencia visual.

Sin embargo, como indican Pellicer et al. (2014), la mayoría de los estudios en esta temática utilizan un Modelo Digital del Terreno (MDT) como superficie de entrada para el cálculo de las cuencas visuales (Bishop y Miller, 2007). Sus resultados pueden ser imprecisos en áreas con presencia de edificios, ya que al utilizar sólo el MDT pueden ser consideradas áreas visibles, cuando en realidad no lo son (Riggs y Dean, 2007). Además, pocos estudios (Bishop et al., 2000; Sander y Manson, 2007; Riggs y Dean, 2007) han considerado el efecto de esas construcciones debido a la escasa disponibilidad y a la dificultad de obtener datos sobre ellos (Pellicer et al., 2014).

El objetivo de este trabajo fue comparar el resultado de las líneas de horizonte obtenidas desde un mismo punto de observación a partir de dos métodos de cálculo diferentes. El Método 1 utilizó el Modelo Digital de Superficie (MDS) del LIDAR combinado con la delimitación de huella de edificios según Catastro. El Método 2 utilizó el atributo de altura de edificios proveniente del Catastro sobre el MDT como referencia. En ambos casos tratamos de generar modelos tridimensionales del área urbana de estudio, fácilmente reproducible y 
actualizable, para anticipar los posibles menoscabos que pudieran ocasionarse a consecuencia de las variaciones volumétricas de los edificios dentro de los entornos consolidados. En los últimos años, se ha producido un aumento y liberalización de importantes recursos de información geográfica que requieren la puesta a punto de métodos y estrategias para su procesado, así como criterios para su valoración. Los dispositivos LIDAR (Light Detection and Ranging) son un claro ejemplo de esta evolución. Su utilización se da en diferentes campos: urbanismo, hidrología, medio ambiente y, por supuesto, en el turístico, como síntesis en buena medida, de todos los anteriores. Por su parte, la Dirección General del Catastro, organismo dependiente del Ministerio de Economía, al menos desde la implantación catastral de los años setenta, se inició en la tarea de inventariar, con grandes escalas, todos los inmuebles del país, tanto de rústica como de urbana. Con estas fuentes es posible modelizar de forma tridimensional el espacio abarcando tanto la superficie geográfica como el arbolado o la propia edificación, abriendo la posibilidad a múltiples tipos de análisis.

\section{Desarrollo}

\section{El uso de archivos LIDAR para la modelización urbana}

Uno de los principales retos en la tecnología LIDAR es la utilización de un adecuado algoritmo de filtrado en la clasificación de los puntos que permita trabajar con ellos en función de su naturaleza. Este proceso de clasificación es el trabajo más importante de edición de una nube de puntos LIDAR (Arranz et al, 2012). La clasificación dará lugar a conjuntos de puntos según tipología: puntos pertenecientes a suelo desnudo, superficies de agua, vegetación, edificación, ruido, líneas eléctricas, etc. Estos datos contienen información de las coordenadas de los puntos donde se producen las reflexiones, tanto si se produce en el suelo como en cualquier objeto que sobresalga de la superficie terrestre, como es el caso de los edificios (Estornell et al., 2011). Cuando se desea registrar información procedente de un entorno urbano, uno de los principales objetivos perseguidos es la documentación del área edificada. Así, a partir de datos LIDAR se pueden generar productos de alta resolución espacial como un MDT y un MDS (Estornell et al., 2011).

Tradicionalmente, este trabajo estaba reservado a la restitución fotogramétrica que realizaba el registro midiendo los límites de los aleros de las edificaciones. El uso del LIDAR permite obtener una densa nube con puntos sobre los aleros de los edificios, sus fachadas, así como puntos sobre el suelo, la vegetación, e incluso, los vehículos y los elementos del mobiliario urbano. Por tanto, considerando el mismo objetivo de extraer el área edificada de un entorno urbano, se precisan algoritmos que permitan diferenciar puntos LIDAR tomados sobre edificaciones del resto de puntos.

En esta investigación hemos trabajado con los archivos LIDAR disponibles en formato. LAZ de $2 \times 2$ km de extensión (densidad de 0,5 puntos /m2) que ofrece el del Instituto Geo- 
gráfico Nacional (IGN) dentro de su Plan Nacional de Ortofotografía Aérea (PNOA). Las especificaciones Técnicas para el desarrollo de las nubes de puntos LIDAR de la Comunidad Autónoma de Canarias (DG. IGN, 2016), señalan que se ha empleado el estándar de clasificación del American Society for Photogrammetry and Remote Sensing (ASPRS). Dicha clasificación se realiza de forma automática en los ficheros LAS ajustándose a los siguientes parámetros:

- Suelo (clase 2)

- Vegetación baja (0-1,5 m), media $(1,5$ a3,0 m) o alta $(>3,0)$ clases 3, 4 ó 5, respectivamente

- Edificios (clase 6)

- Solape (clase 12)

- Sin clasificar: puntos correspondientes a vegetación o edificios sin clasificar (clase 1)

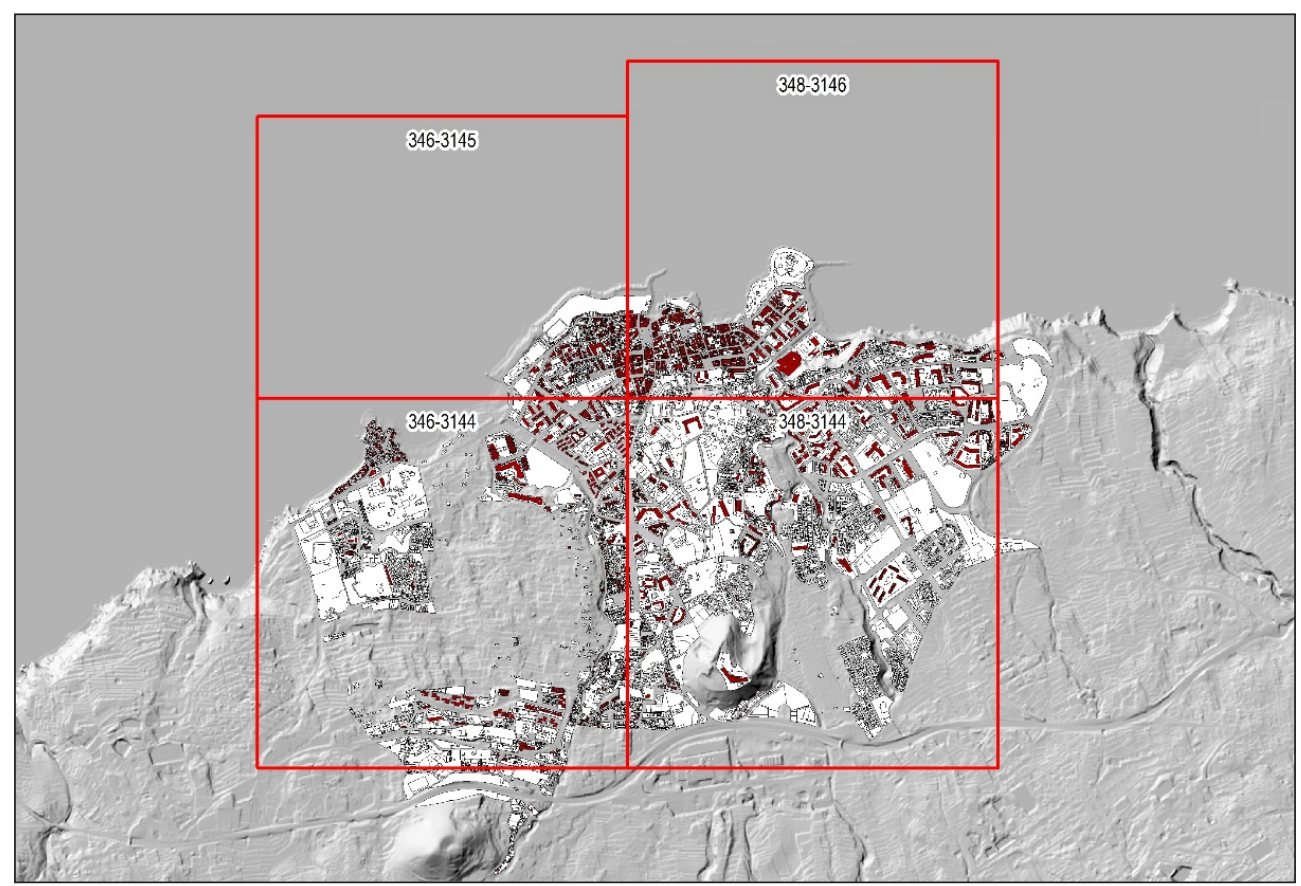

Figura 1. Encuadre del núcleo turístico del Puerto de la Cruz (Tenerife) y posición de las 4 cuadrículas LIDAR empleadas en el análisis. Fuente: elaboración propia. 
Limitaciones en los resultados de la formación de MDS a partir de LIDAR

Para el desarrollo de esta investigación se ha utilizado el software ArcGis Desktop en su versión 10.5. Para el área urbana del Puerto de la Cruz se ha procedido a trabajar con las cuatro hojas $(346-3145 ; 348-3146 ; 346-3144 ; 348-3144)$ de $2 \times 2 \mathrm{~km}$ que abarcan el núcleo central turístico del municipio (Figura 1). Dichas hojas reúnen algo más de 18 millones y medio de puntos. Una vez preparada la información se procedió a transformar los archivos. laz en archivos.las, mediante la herramienta auxiliar LasTools.

Una vez implementado la información en una geodatabase obtuvimos una primera estadística y previsualización de los datos. Esta permitió identificar una clasificación inexacta de la nube de puntos. El algoritmo de clasificación automática que emplea el IGN en sus archivos detectó un 6,7\% de los puntos clasificados como edificación, descartando otros que son igualmente identificativos de este tipo de usos. Como se puede apreciar en la Tabla 1, se trata de un error común entre todas las hojas que se deriva de la trayectoria de alguna de "las pasadas" para la captura de datos, pues es posible apreciar con claridad unas bandas donde se concentran los errores (Estornell, y Ruiz, 2018).

\begin{tabular}{|c|c|c|c|c|c|c|c|c|c|c|c|}
\hline \multicolumn{7}{|l|}{ Devoluciones } & \multicolumn{5}{|c|}{ Atributos } \\
\hline Devolver & \multicolumn{2}{|c|}{ Conteo de ... } & $\%$ & Z Mín. & Z Máx. & $\wedge$ & \multicolumn{2}{|c|}{ Nombre } & Mín. & Máx. & $\wedge$ \\
\hline $1^{0}$ & \multicolumn{2}{|c|}{18.551 .370} & 96,07 & $-10,84$ & 2049,26 & & \multicolumn{2}{|c|}{ No. de retorno } & 1 & 4 & \\
\hline $2^{\circ}$ & \multicolumn{2}{|c|}{737.848} & 3,82 & $-17,29$ & 631,79 & & \multicolumn{2}{|c|}{ Intensidad } & 0 & 255 & \\
\hline 30 & \multicolumn{2}{|c|}{20.060} & 0,10 & $-27,82$ & 625,78 & & \multicolumn{2}{|c|}{ Código de clase } & 2 & 7 & \\
\hline 40 & \multicolumn{2}{|c|}{625} & 0,00 & $-214,29$ & 598,44 & & \multicolumn{2}{|c|}{ Escanear áng... } & $-39,000$ & 29,000 & \\
\hline Último & \multicolumn{2}{|c|}{18.547 .313} & 96,05 & $-214,29$ & 2049,26 & & \multicolumn{2}{|c|}{ Datos del usu... } & 1 & 1 & \\
\hline Simple & \multicolumn{2}{|c|}{17.809 .467} & 92,23 & $-10,84$ & 2049,26 & & \multicolumn{2}{|c|}{ Origen del pu... } & 45 & 146 & \\
\hline Primero de ... & \multicolumn{2}{|c|}{741.903} & 3,84 & $-6,30$ & 635,71 & $\checkmark$ & \multicolumn{2}{|c|}{ Rojo } & 0 & 65280 & $\checkmark$ \\
\hline \multicolumn{12}{|c|}{ Códigos de clasificación } \\
\hline \multicolumn{2}{|l|}{ Clasificación } & Conte & eo de pu... & $\%$ & Z Mín. & \multicolumn{2}{|c|}{ Z Máx. } & Intensid... & Intensid... & Conteo ... & \\
\hline \multicolumn{2}{|c|}{2 Terreno } & & 4.203 .038 & 21,77 & $-4,71$ & \multicolumn{2}{|c|}{248,78} & 0 & 255 & 0 & \\
\hline \multicolumn{2}{|c|}{3 Vegetación baja } & & 3.128 .978 & 16,20 & $-3,81$ & \multicolumn{2}{|c|}{248,92} & 0 & 255 & 0 & \\
\hline \multicolumn{2}{|c|}{4 Vegetación media } & & 5.314 .540 & 27,52 & $-3,67$ & \multicolumn{2}{|c|}{250,50} & 0 & 255 & 0 & \\
\hline \multicolumn{2}{|c|}{5 Vegetación alta } & & 5.364 .442 & 27,78 & 1,55 & \multicolumn{2}{|c|}{265,51} & 0 & 255 & 0 & \\
\hline \multicolumn{2}{|c|}{6 Edificio } & & 1.292 .444 & 6,69 & 2,04 & \multicolumn{2}{|c|}{251,63} & 0 & 255 & 0 & \\
\hline \multicolumn{2}{|l|}{7 Ruido } & & 6.461 & 0,03 & $-214,29$ & \multicolumn{2}{|c|}{2049,26} & 0 & 255 & 0 & \\
\hline
\end{tabular}

Tabla 1. Resumen estadístico de archivos LIDAR empleados en el análisis. Fuente: elaboración propia. 


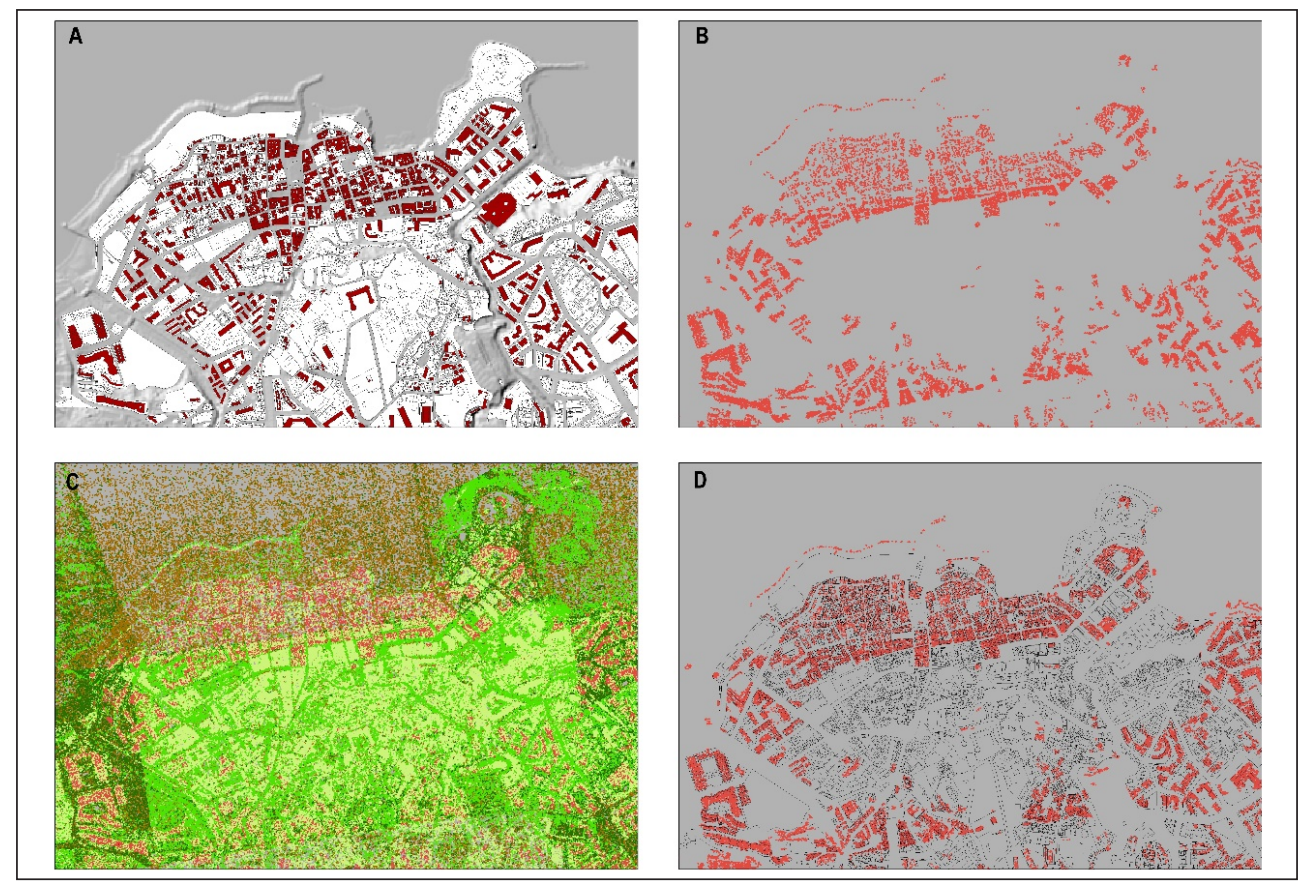

Figura 2. A. Cartografía catastral sobre MDT; B. Nube de puntos clasificados como edificación (valor 6); C. Nube de puntos por clases; D. Nube de puntos de edificación sobre huella catastral de edificios. Fuente: elaboración propia.

Los puntos que dan forma a la edificación están situados en la categoría 5 (vegetación alta) en lugar de la 6 (edificación). Esta mezcla en la categoría clave de edificación impide disponer de un MDS, que pueda ser comparado directamente con la otra técnica de modelización que empleemos.

Para solventar este comportamiento imprevisto de la fuente de información, se procedió a usar un método auxiliar. Este nos permitió finalmente filtrar los puntos que componen la altura de los edificios. En el siguiente punto se expone dicho método.

Obtención de información de elevación a partir de la huella de los edificios y archivos LIDAR.

Si bien contamos con archivos.las capaces de informarnos sobre la altura de cualquier elemento de la superficie del terreno analizado, hemos constatado la imposibilidad inicial de separar la información de los edificios respecto a la vegetación alta. Sin embargo, se dispone de la "huella" o planta de apoyo sobre el suelo de los edificios. Se trata de una información de fácil acceso descargable desde la Sede Electrónica del Catastro y definida a partir de la cartografía catastral elaborada por la Dirección General del Catastro (SEC). 
Con esta información, podemos hacer un filtro espacial para "acotar" los puntos coincidentes con la huella de los edificios, descartando los que no correspondan con dicha posición. Para ello el primer paso será generar un conjunto de puntos de muestra aleatorios para cada huella de edificio. En nuestro caso hemos usado la herramienta de geoprocesamiento "Crear puntos aleatorios" para acotar los conjuntos de puntos representativos de la altura de cada polígono. Con el conjunto de puntos obtenidos para cada identificador de objeto, podremos estimar la altura media de cada uno de los contornos reflejados en la planta del edificio. El resultado obtenido es una nueva entidad que contiene un grupo de puntos para cada edificio.

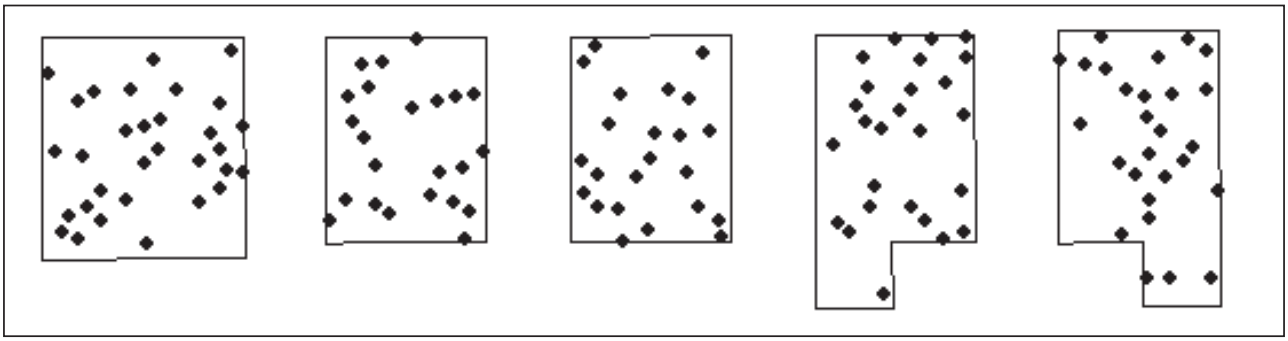

Figura 3. Esquema de asignación aleatoria de puntos a través a la huella de los edificios según cartografía del Catastro. Fuente: elaboración propia.

El siguiente paso que seguimos es proceder a asignar la información de alturas, procedente de la superficie de elevación ráster de primer retorno del LIDAR. Para añadir dicha información se emplea la herramienta de geoprocesamiento "Agregar información de superficie". Con la herramienta de "Resumen de estadística" se trata de generar un único valor de altura para cada edificio. El método estadístico utilizado ha sido el de la media (mean), que proporciona la altura media en metros del tejado de los edificios. Con posterioridad, se procedió a situar la base de extrusión de cada edificio a través del MDT, con paso de malla de 5 metros obtenidos por interpolación a partir de la clase terreno de vuelos LIDAR del Plan Nacional de Ortofotografía Aérea (PNOA). Una vez se obtuvo la cota de planta de cada edificio, se procedió a extrudir la altura de cada edificio en función del atributo obtenido anteriormente. A este método de trabajo lo hemos denominado Método 01 (Figura 4)

\section{El uso de archivos del Catastro para la modelización urbana}

El procedimiento para obtener un modelo urbano tridimensional a partir de la cartografía catastral supone un ejercicio sencillo. El principal problema fue la transformación de la nomenclatura de alturas de edificios propuesta por la Norma Técnica del Catastro. La cartografía catastral se ha representado tradicionalmente a nivel de atributo de las subparcelas o construcciones, la volumetría de la edificación mediante números romanos, indicando el 
número de plantas que tiene un edificio, incluso diferenciando aquellas que se encuentran bajo rasante, mediante una sintaxis de concatenación de valores negativos y positivos (Virgós y Olivares, 2006). Toda esta información recogida en la cartografía como atributo de recintos y dentro de un sistema de información geográfico en formato de vectorial se puede modelizar en 3D. Para ello es necesario analizar el valor del atributo de cada recinto y aplicar un algoritmo para transformarlo a un valor numérico que represente el número de plantas del edificio que se encuentran sobre la rasante del terreno. Este valor se multiplicará por 3 metros, como media bastante real de lo que puede suponer la altura de una planta de un edificio, para representar la altura total de cada volumen; se trata de una aproximación, ya que sabemos que muchos edificios (hoteles, iglesias, centros comérciales, equipamientos, etc.) cuentan con plantas bajas de mayor altura que a la planta tipo. En nuestro caso, hemos procedido de esta forma desarrollando un algoritmo en Python $^{2}$ y tras situar la cota de altura del terreno a través del mismo MDT que en el método anterior, se ha extruido las alturas obteniendo el escenario tridimensional. Este método de trabajo lo hemos denominado Método 02 (Figura 4).

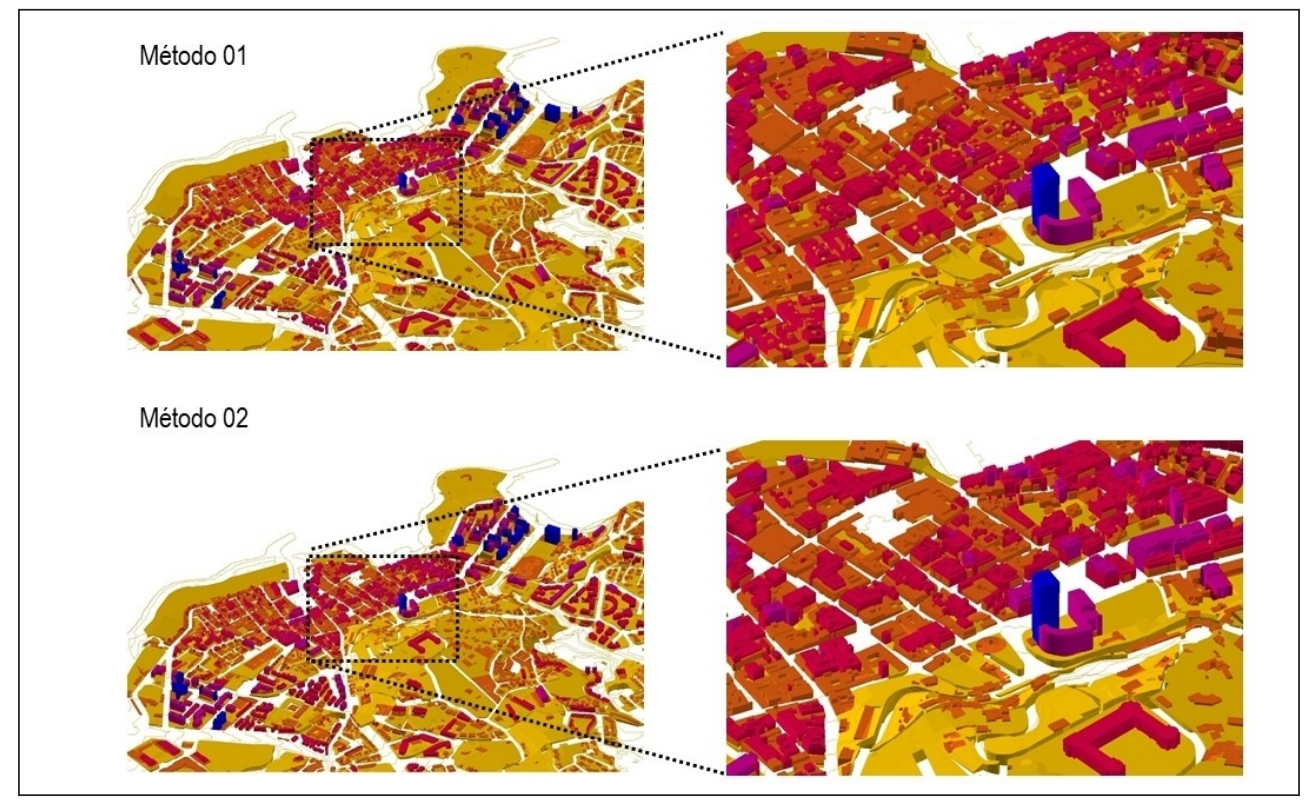

Figura 4. Escenario urbano tridimensional. Método 1: Obtención de alturas a través de LIDAR y huella de edificios según Catastro con MDT. Método 2: Obtención de alturas a través del atributo de altura del Catastro con MDT. Fuente: elaboración propia.

2 Puede ser descargable y comprobable en: https://www.youtube.com/watch?v=90vc8AsdT9k 


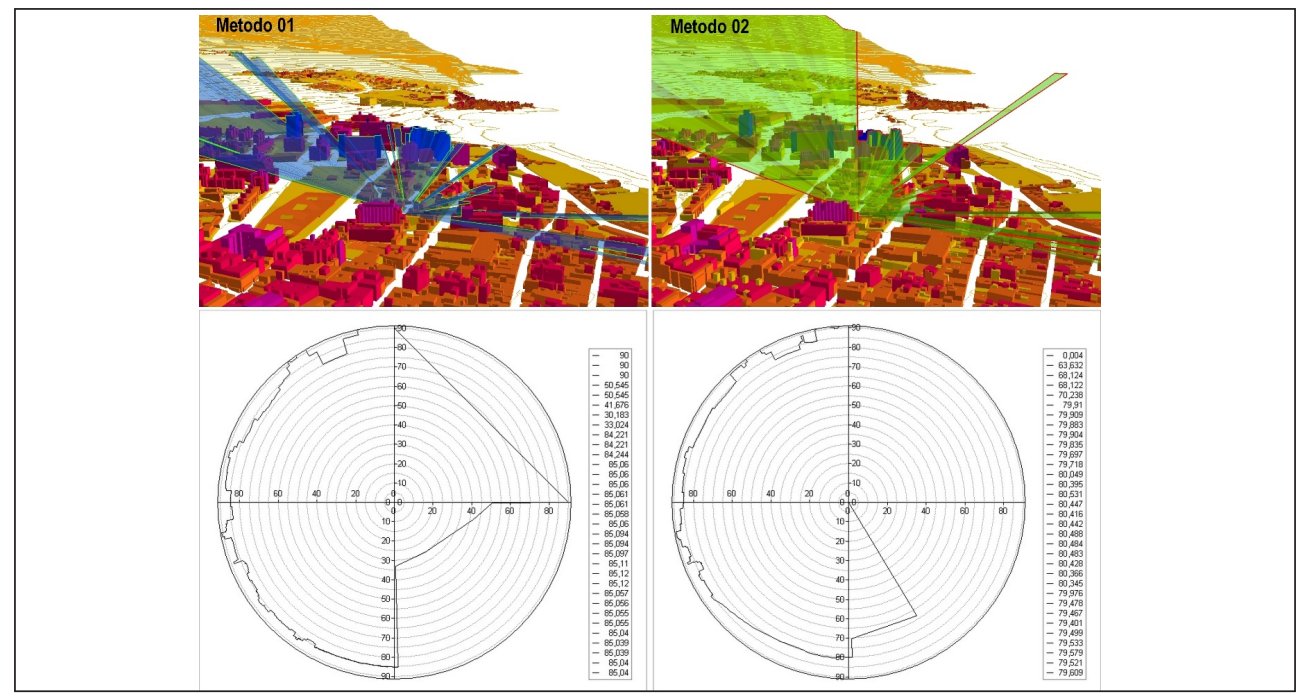

Figura 5. Valoración de la línea de horizonte desde el mismo punto de observación mediante Método 1: Obtención de alturas a través de LIDAR y huella de edificios según Catastro con MDT y Método 2: Obtención de alturas a través del atributo de altura del Catastro con MDT. Gráfico de horizonte desde el punto de observación mediante los dos métodos empleados. Fuente: elaboración propia.

\section{DiscUSIÓN Y CONCLUSIONES}

A partir de los dos modelos obtenidos se ha realizado una evaluación de la línea de horizonte mediante varios puntos significativos del área turística seleccionada. Se han seleccionado quince puntos de observación que se han repartidos por el Puerto de la Cruz, buscando la mayor representatividad de ambientes y morfologías presentes. Una vez seleccionado dichos puntos, se procedió a realizar un análisis de horizonte o de silueta para cada punto y para cada escenario mediante la herramienta 3D Analyst (Método 1 y Método 2). Como ejemplo mostramos una de las valoraciones de la línea de horizonte tomadas en cada escenario desde el mismo punto de vista, acompañándolo de un gráfico polar de horizonte que representa la proporción de cielo visto y las barreras de los edificios y topografía. El gráfico polar representa la visión de una persona ubicada en el punto de análisis que contempla el cielo. Se trata de una representación similar a una fotografía de ojo de pez que se puede usar para representar el grado de proximidad con respecto al punto central de un observador Las áreas interiores (desde el centro hasta el perímetro dibujado) representan los puntos en los que los edificios se extienden en el espacio del cielo. Cuanto más alto es el edificio, más se adentra hacia el centro del círculo.

Como se puede observar en la Figura 5, los resultados obtenidos mediante los dos métodos de modelización no son iguales. De hecho, existen notables diferencias entre ambos. La dife- 
rencia se centra en la interpretación de la altura. Así, con el primer método (Huella de Catastro + LIDAR), empleando la altura media de los recintos a partir de la primera devolución de los puntos del LIDAR, se obtuvo un resultado mucho más preciso y ajustado que el obtenido a partir del método que utiliza las alturas del Catastro. Con el Método 2 (Alturas según Catastro), el error cometido en la utilización de la altura entre forjados fija de tres metros ${ }^{3}$ produjo errores en la elevación de los alzados edificados, generando una percepción artificial de las líneas de horizonte. El conjunto de este trabajo mejoraría su calidad y rigor si incorporara puntos de muestreo para el análisis in situ de la visibilidad en cada uno de ellos, contrastando los resultados con los 2 métodos de estudio empleados (Pellicer et al., 2014). La precisión de esta fase no se ha alcanzado, teniendo sólo una constatación visual de la calidad del Método 1.

La importancia de los datos en los procesos de planificación y gestión de las áreas turísticas de litoral se justifica por sus características propias de la actividad turística, configurándolos como intensivos en información y, sobre todo, conocimiento. En este contexto, las tecnologías de la información y la comunicación, en general, y de las de análisis espacial, en particular, se convierten en un factor de competitividad fundamental (Buhalis y Matloka, 2013) y en instrumentos básicos para generar soluciones inteligentes.

En este trabajo se ha tratado de valorar en qué medida las nuevas fuentes de información y las nuevas tecnologías de análisis espacial pueden ser útiles a propósito de previsualizar con antelación, los escenarios urbanos con suficiente precisión y calidad permitiendo valorar los perjuicios que, en su caso, una determinada operación urbanística podría provocar, en relación con la ocultación de vistas o efecto pantalla de un edificio respecto a otro. En este sentido, la técnica desarrollada en este trabajo puede ser de utilidad para ayudar los procesos de toma decisiones en la cada vez más generalizada puesta en valor de las azoteas de los establecimientos hoteleros, a través de su instalación de piscinas con solarium, bares, restaurantes, terrazas, etc.

La existencia de herramientas que en ese sentido asistan a la planificación sobre la usurpación de vistas y paisaje y las repercusiones que estas pudieran producir, son posibles y se afirma el interés en seguir profundizando en ellas. Así mismo, su empleo no se limita a la valoración de escenarios dentro de un contexto de recursos y conflictos jurídicos, sino que puede emplearse en una versión "positiva" como asistente en materia de valoración del potencial de remanente de vistas en un destino. Como línea de trabajo futura se apunta valorar las posibilidades que ofrecen un software como CityEngine (ESRI) para la gestión de esta información ya que ofrecen entornos y herramientas predefinidas ágiles y fáciles de actualizar (Temes \&Moya, 2019).

Si bien se partía de la hipótesis de que la tecnología LIDAR por su calidad y precisión sería sin lugar a dudas la mejor solución, se constatan un error de clasificación automática de los puntos que obligan al empleo de un método intermedio. El Método 01 en el que se combina la huella de la edificación junto con la altura media a partir de los archivos LIDAR dan el mejor resultado para los 15 puntos de control analizados en el estudio.

3 En otros trabajos como los de Pellicer et al. (2014) se usa una altura media de 2,75. 


\section{Agradecimientos}

La investigación que da lugar a este trabajo constituye uno de los resultados del proyecto "Análisis de la sostenibilidad urbana como estrategia de regeneración del espacio público de las áreas turísticas de litoral", financiado por la Fundación CajaCanarias. Asimismo, se inscribe en el proyecto "Overtourism in Spanish Coastal Destinations. Tourism Degrowth Strategies" [RTI2018-094844-B-C31] del Ministerio de Ciencia, Innovación y Universidades.

\section{Bibliografía}

Antón-Clavé, S. (1998): La urbanización turística. De la conquista del viaje a la reestructuración de la ciudad turística. Documents d'Anàlisi Geogràfica, 32, 17-43.

Arranz, J.J., Ormeño, S. y Vicent, J.M. (2012): Algoritmo para la clasificación de nubes de puntos LiDAR en entornos urbanos: discriminación entre vegetación y edificaciones. En I Congreso Iberoamericano de Geomática y Ciencias de la Tierra. X Topcart, Madrid.

Bishop, I.D., Wherrett, J.R., Miller D.R. (2000): Using image depth variables as predictors of visual quality. Environment and Planning B: Planning and Design, 27(6), 865-875.

Buhalis, D. y Matloka, J. (2013): Technology-enabled Tourism Destination Management and Marketing. En Costa, C.; Panyik, E. y Buhalis, D. (eds.). Trends in European tourism planning and organisation. Buffalo, NY: Channel View Publications, 339-350.

Cavailhès, J., Brossard, T., Foltête, J.C., Hilal, M., Joly, D., Tourneux, F.P., Tritz, C., Wavresky, P., (2009): GISbased hedonic pricing of landscape. Environmental and Resource Economics, 44(4), 571-590.

Celdrán-Bernabeu, M.A.; Mazón, J.-N. y Giner, D. (2018): Open Data y turismo. Implicaciones para la gestión turística en ciudades y destinos turísticos inteligentes. Investigaciones Turísticas 15, 49-78. http://dx.doi.org/10.14198/INTURI2018.15.03

Cortina, F., Izquierdo, M., Prado, J. y Velasco, M. (2016): Uso de información complementaria en las estadísticas de turismo. Revista Índice, 25-28.

Danese, M.; Nolè, G. y Murgante, B. (2009): Visual impact assessment in urban planning. Environ Earth Sci, vol. 176, 133 - 146.

Danese, M.; Nolè, G. y Murgante, B. (2011): Identifying viewshed: new approaches to visual impact assessment. Studies in computational intelligence, vol. 348, 73 - 89.

De Aranzabal, I., Schmitz, M. F. y Pineda, F. D. (2009): Integrating landscape analysis and planning: A multi-scale approach for oriented management of tourist recreation. Enviromental Management, 44 (5), 938-951.

Dirección General del Instituto Geográfico Nacional (2016): Especificaciones Técnicas para el contrato de suministro de una base de datos especializada de nubes de puntos LiDAR de la Comunidad Autónoma de Canarias

Dos-Santos, P. (2011): Marco teórico-metodológico de los estudios de paisaje. Perspectivas de aplicación en la planificación del turismo. Estudios y Perspectivas en Turismo 20, 522-541. 
Estornell, J., Ruiz, L. (2018): Generación de MDE Y MDS en una zona de vegetación arbórea y arbustiva mediante datos Lidar e imágenes de Satélite Quickbird.

García Sanjuán, L. (2006): Análisis de pautas de visibilidad en la distribución de monumentos megalíticos de Sierra Morena Occidental. En Grau Mira (ed.) La aplicación de los SIG en la Arqueología del Paisaje. Alicante, 122-157.

Gupta, V.; Mythili, G. \& Hedge D.S. (2009): Deriving Implicit Prices for Urban Environment Amenities from Mumbai Housing Prices: A Revealed Preference Approach. En European Consosrtium for Landscape Economics y Universidad de Viena BOKU (org.) Conferencia Internacional en Economías del Paisaje. Viena. 248-255.

Hamilton, S.E., Morgan, A. (2010). Integrating LiDAR, GIS and hedonic price modeling to measure amenity values in urban beach residential property markets.Computers, Environment and Urban Systems, 34(2), 133-141.

Hermosilla, T., Ruiz, L.A., Fdez. Sarriá, A., Recio, J.A, (2009): Detección automática de edificios en imágenes aéreas mediante técnicas de teledetección y análisis contextual. En XIII Congreso de la Asociación española de Teledetección, Cataluña.

Ivars, J.; Solsona, J.; Giner, D. (2016): Gestión turística y tecnologías de la información y la comunicación (TIC): El nuevo enfoque de los destinos inteligentes. Documents d'Anàlisi Geogràfica, vol. $62 / 2327-346$.

Jordan, V. (2015): Aplicación de datos LiDAR del sistema aéreo en la actualización catastral urbana. Proyecto Final de Carrera inédito. http://hdl.handle.net/10251/55006.

Legget, C. y Bockstael, N.E. (1999): Evidence of the Effects of Water Quality of Residencial Land Prices. Journal of Environmental Economics and Management, 39, 21-144

Llobera, M. (2003): Extending GIS-based visual analysis: the concept of visualscape. International Journal of Geographical Information Science, 17: 25-48.

More, T.A., Stevens, T. y Allen P.G (1988): Valuation of urban parks. Landscape and Urban Planning, $15,139-152$.

Moya, L.; Fernández, C.; Escamilla, F. (2017): Evolución del tamaño de la vivienda de promoción pública y su comparación con el resto del parque residencial construido en Madrid entre 1940-2010. Informes de la Construcción, 69 (545). doi: http://dx.doi.org/10.3989/ic.16.040.

Otero, I.; Varela, E.; Mancebo, S. y Ezquerra, A. (2009): El análisis de visibilidad en la evaluación de impacto ambiental de nuevas construcciones. Informes de la Construcción, vol. 61, 515, 67 - 75.

Pellicer, I., Estornell, J., Martí, J. (2014): Aplicación de datos LiDAR aéreo para el cálculo de cuencas visuales. Revista de Teledetección, 41, 9-18.

Pérez, J.L; Delgado, J. (2015): Extracción Automática de Edificios a partir de Modelos Digitales de Superficies: el sistema BUILDING. Espacio Tiempo y Forma. Serie VI, Geografía, [S.1.], n. 1, ene. 2008. ISSN 1130-2968. Disponible en: doi:https://doi.org/10.5944/etfvi.1.2008.1457.

Peñarrubia, M. P. (2018): Los datos estadísticos públicos y su uso en el conocimiento del comportamiento de los turistas en destinos inteligentes. Universitat de València. Tesis doctoral inédita. 
Riggs, P.D., Dean, D.J. (2007): An Investigation into the Causes of Errors and Inconsistencies in Predicted Viewsheds. Transactions in GIS, 11(2), 175-196.

Sander, H.A. y Manson, S.M. (2007): Heights and locations of artificial structures in viewshed calculation: how close is close enough. Landscape and Urban Planning 82(4), 257-270.

Santos-Pavón, E.; Fernández-Tabales, A.; Muñoz-Yules, O. (2016): La incorporación del paisaje a la planificación turística. Análisis de la estrategia de turismo sostenible de Andalucía. Cuadernos de Turismo, 37, 175-202.

Santos-Preciado, J. (2015): Metodología para medir la estructura urbana de la ciudad actual, utilizando la base de datos del catastro. Aplicación al sector suroeste de la Comunidad de Madrid. Boletín de la Asociación de Geógrafos Españoles, 0 (67). doi:http://dx.doi.org/10.21138/bage.1816

Santos-Pérez, L (2015): La tecnología LIDAR en el procedimiento de regulación catastral. Resultado de su validación. Revista CT-Catastro, 83, 31-51

Schultz, T. y Waltert, F. (2009): How local landscape resources affect property prices: Evidence from an hedonic pricing model". En European Consosrtium for Landscape Economics y Universidad de Viena BOKU. Conferencia Internacional en Economías del Paisaje. Viena. 314-327.

Temes, R. (2008): Las fuentes catastrales y la identificación de las transformaciones en los tejidos urbanos. Revista CT-Catastro, 64, 55-78. http://hdl.handle.net/10251/11114

Temes, R. y Moya, A. (2019): CityEngine. Manual práctico. Editorial Ra-Ma, Madrid

Torres, E. y Navarro, E. (2007): La congestión urbanística como factor reductor de la calidad turística y de vida en los destinos maduros, Estudios Turísticos, 172-173, 193-199.

Vera, F.; López, F.; Marchena, M. y Antón, S. (2011): Análisis territorial del turismo y planificación de destinos turísticos. Editorial Tirant Lo Blanch, Valencia.

Villar, A. (2013): La mercantilización del paisaje litoral del mediterráneo andaluz: El caso paradigmático de la Costa del Sol y los campos de golf. Revista de Estudios Regionales, 96, 215-242.

Virgós, L. y Olivares, JM. (2006). Catastro 3D en Internet.

Cómo citar este artículo:

Temes Cordovez, R., Simancas Cruz, M., Peñarrubia Zaragoza, M. P., García Amaya, A. (2019). Aplicación de modelos digitales a la determinación del valor de vistas al mar en las áreas turísticas de litoral. Cuadernos de Geografía, 103, 7-24.

https://doi.org/10.7203/CGUV.103.15560

\section{(c) $(1) \Theta \Theta$}

Este obra está bajo una licencia de Creative Commons Reconocimiento-NoComercial-SinObraDerivada 4.0 Internacional. 